\title{
Relative difference in muscle strength between patients with knee osteoarthritis and healthy controls when tested bilaterally and joint-inclusive: an exploratory cross-sectional study
}

\author{
K. Vårbakken ${ }^{1 *}$, H. Lorås ${ }^{2}$, K. G. Nilsson ${ }^{3}$, M. Engdal ${ }^{4}$ and A. K. Stensdotter ${ }^{1}$
}

\begin{abstract}
Background: To improve the goal-directedness of strength exercises for patients with knee osteoarthritis (KOA), physical rehabilitation specialists need to know which muscle-groups are most substantially weakened across the kinetic chain of both lower extremities. The purpose was to improve the knowledge base for strength exercise therapy. The objective was to explore the relative differences in muscle strength in the main directions bilaterally across the hip, knee, and ankle joints between patients with light-to-moderate symptomatic and radiographic KOA and people without knee complaints.

Methods: The design was an exploratory, patient vs. healthy control, and cross-sectional study in primary/ secondary care. Twenty-eight patients with mild to moderate KOA (18 females, mean age 61) and 31 matched healthy participants (16 females, mean age 55), participated. Peak strength was tested concentrically or isometrically in all main directions for the hip, knee, and ankle joints bilaterally, and compared between groups. Strength was measured by a Biodex Dynamometer or a Commander II Muscle Tester (Hand-Held Dynamometer). Effect sizes (ES) as Cohen's $d$ were applied to scale and rank the difference in strength measures between the groups. Adjustment for age was performed by analysis of covariance.

Results: The most substantial muscle weaknesses were found for ankle eversion and hip external and internal rotation in the involved leg in the KOA-group compared to the control-group (ES [95\% Cl] $-0.73[-1.26,-0.20]$, $0.74[-1.26,-0.21],-0.71[-1.24,-0.19]$, respectively; $p<0.01)$. Additionally, smaller but still significant moderate muscle weaknesses were indicated in four joint-strength directions: the involved leg's ankle inversion, ankle plantar flexion, and knee extension, as well as the uninvolved leg's ankle dorsal flexion $(p<0.05)$. There was no significant difference for 17 of 24 tests.

Full list of author information is available at the end of the article International License (http://creativecommons.org/licenses/by/4.0/), which permits unrestricted use, distribution, and reproduction in any medium, provided you give appropriate credit to the original author(s) and the source, provide a link to the Creative Commons license, and indicate if changes were made. The Creative Commons Public Domain Dedication waiver (http://creativecommons.org/publicdomain/zero/1.0/) applies to the data made available in this article, unless otherwise stated. 
(Continued from previous page)

Conclusions: For patients with KOA between 45 and 70 years old, these explorative findings indicate the most substantial weaknesses of the involved leg to be in ankle and hip muscles with main actions in the frontal and transverse plane in the kinetic chain of importance during gait. Slightly less substantial, they also indicate important weakness of the knee extensor muscles. Confirmatory studies are needed to further validate these exploratory findings.

Keywords: Osteoarthritis, knee, Healthy volunteers, Muscle strength, Muscle strength dynamometer, Primary health care, Secondary care, Cross-sectional studies [Mesh terms], Exploratory studies [text-word]

\section{Background}

Osteoarthritis (OA) is a leading cause of pain and disability worldwide [1], with knee OA (KOA) exhibiting an incidence of 240 per 100,000 person-years in adults or about 2.5 times that of hip OA [2].

Further known or accepted, governmental-approved guidelines for management in primary care in Denmark and Sweden state that a KOA diagnosis can be made clinically and that strength training and education are among the first-line of care [3]. Diagnostically, this concurs with the criteria of the European League Against Rheumatism's (EULAR) [4] for primary care, on the one hand. For hospital care, on the other hand, much accepted criteria are those from the American College of Rheumatology (ACR) [5]. More recently, KOA is conceptualized as a whole person chronic disease [6], one where symptoms and signs develop over decades [7], and that is manageable for most people by early diagnosis and individualized management [6].

Management by strength exercise therapy demonstrates the largest effect sizes (ES) on pain and function compared to other active therapies in KOA according to systematic reviews and meta-analyses of randomized controlled trials (RCTs) [8-11]. Further, in the most affected leg, systematic reviews of cross-sectional casecontrol studies indicate muscle weaknesses across the hip [12] and the knee muscles [13] for patients with KOA. However, even for the affected leg, there is limited knowledge of the relative difference between ankle, knee, and hip muscle strength in patients with KOA compared to healthy controls. Furthermore, there is particularly limited knowledge regarding ankle strength. Additionally, regarding the (least or) non-affected leg, knowledge about strength deficits and their relative difference is even more limited. Overall, such extensive strength knowledge can potentially lead to improved strategies for strength exercises for patients with KOA.

Thus, to improve the knowledge required for strength exercise therapy, we aimed to explore the relative difference in muscle strength bilaterally in the main directions across the hip, knee, and ankle joints between patients with $\mathrm{KOA}$ and individuals without knee complaints in a cross-sectional study.
More specifically, by application of traceable and reliable strength dynamometers, we performed the first ever full bilateral overview of strength deficits of the main muscle-groups of the lower limb in patients with KOA compared to healthy controls through a well-powered exploratory study $[14,15]$.

\section{Methods}

This study was a part of a larger comprehensive study of functional aspects on knee osteoarthritis, FUNKART.

\section{Design and ethics}

We set out to develop an exploratory [14, 15] crosssectional, age- and gender-matched [16] patient versus healthy control study. The study was approved by a Regional Ethics Committee for Medical and Health Research (the Regional Ethics Committee North, RECnorth 2016/984) and conducted according to the Helsinki declaration and Norwegian laws. All participants received oral and written information and signed an informed consent before entering the study.

We recruited individuals with KOA referred by general physicians (GPs) to private physiotherapy clinics and to the osteoarthritis school at a university hospital from Nov 2016 to Dec 2017. Frequency-matched [16] healthy volunteers were aimed to be recruited from work places in the vicinity of the lab by in-person, physical and electronic communication.

\section{Participants}

The inclusion criteria for patients were having KOA in the tibiofemoral joint (s) of one or both legs diagnosed clinically and verified radiologically (Kellgren-Lawrence grade 1-4) [17], with main problem of pain and limited physical function related to the knee (s) and be symptomatic for $>3$ months and daily during last month.

The inclusion criteria for both groups were being male or female between 45 and 70 years of age, able to understand written and oral Norwegian, and be able to walk on even ground and stairs. Healthy controls had to be without pain or knee complaints during common activities of daily life. 
The exclusion criteria for all participants were surgery to a lower extremity $<3$ years ago, prior lower limb fractures, generalized pain, pain from the spine, hips, or ankles competing with that from the knee (in the KOAgroup), knee pain (in the control-group), body mass index $>35$, and medical diagnoses other than KOA with clear negative influence on physical function and pain.

\section{Measurements}

\section{Strength measurements}

Before strength testing, the participants warmed up by several performance tests (e.g. the 6-min walk test [6MWT] and the 10-step up-and-down stair-climb test [T10StUpDwT]) and a set of 15 repetitions at low-tomoderate load on each specific exercise. Strength or concentric peak torque were recorded at $60 \%$ by the isokinetic mode applying the Biodex ${ }^{\circ}$ System 4 Dynamometer (Biodex Medical Systems, NY, USA) $[18,19]$. With the participants sitting, we sequentially tested strength with the back rest tilted $70^{\circ}$ off the horizontal line in the following order: knee flexion and extension, hip internal and external rotation, ankle inversion and eversion, and ankle plantar and dorsal flexion. With the participants supine, we tested hip extension and flexion. These positions and setups were according to the Biodex manual [20], except for the hip rotation tests that were performed according to Baldon et al. [21]. Finally, with the participants supine on a therapy bench, we tested isometric hip abduction and adduction strength with the hips in a neutral ab-adducted position, applying a handheld muscle tester dynamometer (HHD) according to Thorborg et al. (2013) [22]. Specifically, the HHD (Commander Muscle Tester, JTech Medical Industries, Utah, USA) was placed under a non-elastic fixation belt (art. no. 304018, Fysiopartner, Norway) that was looped around the epicondyle of the femur and a vacuum pump [that was fastened on the wall] (art. no. 071458045, Würt, Germany). Before testing, the pelvis was secured bilaterally against inferior and lateral displacement according to Vaarbakken and Ljunggren [2007] [23].

We applied five consecutive maximum strength tests by the Biodex system and three repeated trials by the belt-fixed HHD. Oral encouragements were applied according to principles in Thorborg (2013) [22]. For the knee tests, the Biodex system's "passive isokinetic mode" was chosen, to better accommodate eccentric performance (eccentric data not reported here). Accordingly, fully passive recordings were taken to correct for gravity (see Data processing). The other tests by the Biodex system, we corrected for gravity by its software. The Biodex system was calibrated before each session according to the manual [20]. The HHD is certified by the National Institute of Standards and Technology (NIST) standards. The latter device is self-calibrating and was compared daily to an identical reserve HHD, as both were compared to traceable Olympic Competition Weights [24] (Eleiko, Halmstad, Sweden). The test team trained about $40 \mathrm{~h}$ to execute the complete strength protocol within $1.5 \mathrm{~h}$.

\section{Procedures and supplementary measures}

To enable appropriately judging the background variables (demographics, personal, and clinical factors) and the warm up procedures of the present study, we present below supplementary measures mainly presented in Table 1 (Results) and another study in the present journal [25].

For each participant, questionnaire, functional, and strength data were collected within a period of 2 weeks. The questionnaires were e-mailed as web-surveys together with the informed consent forms by the Infopad system [26]. All participants filled out the self-reported outcome measurement instruments Numeric Pain Rating Scale (NPRS) [27-29] and Knee Injury and Osteoarthritis Outcome Scale (KOOS) [30-32]. KOOS was chosen (over the more widely applied Western Ontario and McMaster Universities Arthritis Index [WOMAC]) due to being free/open access [33], its inclusion of Recreation and Sports and WOMAC, and its knee specificity. In the week thereafter, in the lab, we registered personal or demographic characteristics, degree of radiographic KOA (radiology reports) [17], the 6MWT [34, 35], and the T10StUpDwT [33, 34, 36]. (The latter tests were embedded as a strength warm-up procedure and their results are reported elsewhere [25].) At the end of the lab-session, we measured peak strength. In all the tests, the Biodex "cushion -function" was set at hard and the windowing to $80 \%$. The study's questionnaires took on average $40 \mathrm{~min}$ and the total test protocol $2.7 \mathrm{~h}$ (that is, in the extended or full study protocol).

\section{Data processing}

For concentric knee extensor strength, the passive torques were added to the active ones to correct for the limb's own torque, whereas for concentric knee flexion strength the passive torques were subtracted. Peak knee strength at the $30^{\circ}$ knee flexed position $\left(0^{\circ}=\right.$ straight knee) was reported. The isokinetic mode with automatic gravity correction was used for all but the knee flexion and extension protocol in passive mode where gravity was corrected for afterwards.

For the HHD isometric hip tests, we calculated torque $(\mathrm{Nm})$ by multiplying force $(\mathrm{N})$ by the distance $(\mathrm{m})$ from the top of trochanter major to the femoral lateral condyle. Strength was taken as "best of" or peak torque and divided by body weight, thus we report $\mathrm{Nm} / \mathrm{kg}$. 
Table 1 Background or personal and clinical factors in the case- and control-group

\begin{tabular}{|c|c|c|c|c|c|}
\hline ICF & Variables & $\begin{array}{l}\text { Cases } \\
(n=28)\end{array}$ & $\begin{array}{l}\text { Controls } \\
(n=31)\end{array}$ & $\begin{array}{l}\text { Statistics } \\
t, \times 2, U\end{array}$ & $P$-value \\
\hline \multirow[t]{11}{*}{ Personal factors } & Female, n (\%) & $18(64)$ & $16(52)$ & $379(x 2)$ & 0.3294 \\
\hline & Age, yrs., M (SD) & $61.7(6.4)$ & $55.3(8.0)$ & $3.4(t)$ & $0.0014^{*}$ \\
\hline & Height, m, M (SD) & $1.72(0.10)$ & $1.73(0.09)$ & $-0.7(t)$ & 0.517 \\
\hline & Weight, kg, M (SD) & $82.9(12.7)$ & $80.4(16.6)$ & $0.7(\mathrm{t})$ & 0.517 \\
\hline & $\mathrm{BMI}, \mathrm{kg} / \mathrm{m}^{2}, \mathrm{M}(\mathrm{SD})$ & $24.3(3.5)$ & $25.2(5.1)$ & $1.0(\mathrm{t})$ & 0.317 \\
\hline & Education, n (\%) & & & & \\
\hline & secondary school (10 yrs) & $1(4)$ & $0(0)$ & & \\
\hline & high school (13yrs) & $6(21)$ & $6(19)$ & & \\
\hline & graduate (16yrs) & $14(50)$ & $13(42)$ & & \\
\hline & post graduate (18yrs. +) & $7(25)$ & $12(39)$ & $368(U)$ & 0.281 \\
\hline & Dominant leg (right, left, n) & 26,2 & 28,3 & & \\
\hline \multirow[t]{15}{*}{ Body function } & Years since diagnosis, M (SD) & $10.2(8.6)$ & & & \\
\hline & Years of knee pain, n (\%) & & & & \\
\hline & $1 \mathrm{yrs}$ & $2(7)$ & & & \\
\hline & 1 to 3 yrs. & $3(11)$ & & & \\
\hline & 3 to $10 \mathrm{yrs}$ & $7(25)$ & & & \\
\hline & $>10 y r s$ & $16(57)$ & & & \\
\hline & Pain last week, Med (IQR) & $3.5(4.8)$ & $0.0(1.0)$ & 3.0 & \\
\hline & KOOS Pain, Med (IQR) (R) & $58.8(18.8)$ & $98.4(3.6)$ & -38.9 & \\
\hline & Case-group only & & & & \\
\hline & X-ray grade (n knees, \%) & Inv. leg & Uninv. leg & & \\
\hline & No X-rays taken & $0(0)$ & $10(36)$ & & \\
\hline & KL-grade II & $9(32)$ & $9(32)$ & & \\
\hline & KL-grade III & $17(61)$ & $8(29)$ & & \\
\hline & KL-grade IV & $2(7)$ & $1(4)$ & & \\
\hline & KOOS Sympt., Med (IQR) (R) & $58.9(33.9)$ & $96.4(7.1)$ & -35.8 & \\
\hline \multirow[t]{2}{*}{ Activity function. } & KOOS ADL, Med (IQR) (R) & $66.7(39.6)$ & $100.0(13.3)$ & -32.4 & \\
\hline & KOOS Sport/Rec, Med (IQR) (R) & $30.0(25.6)$ & $100.0(5.0)$ & -65.0 & \\
\hline Participation function & KOOS QoL, Med (IQR) (R) & $43.8(25.0)$ & $100(13.9)$ & -56.2 & \\
\hline
\end{tabular}

Notes: Bold font and * = highly significant different variable; KOOS Knee Injury and Osteoarthritis Outcome Scale, 0 to 100 , worst to best; Sympt. = symptoms (a KOOS subscale); $A D L$ Activity in daily life, Sports $/$ Rec $=$ Sports and Recreation; $\mathrm{t}=$ Independent $\mathrm{t}$-test statistics; $\mathrm{X} 2=\mathrm{Chi}$-square test statistics; $\mathrm{U}=\mathrm{Mann}$-Whitney $\mathrm{U}$ test statistics. KL-grade: Kellgren-Lawrence osteoarthritis grade

\section{Statistical analysis}

A pilot-based sample size calculation, based on unpublished lab-data from an osteoarthritis study in the same area [37], with $\alpha$ (two-tailed) of $0.05, \beta 0.20$, SD 0.7, and moderate effect size of 0.64 , gave the estimate that 20 participants was needed per group. However, due to the explorative design [15], we aimed for 30 participants in each group.

Normality was inferred by histogram inspections, Normal P-P plots, and Kolmogorov-Smirnov tests. For the equal variance assumption, Levene's test were performed. For parametric strength data with no significant outliers and equal variance, a one-factor (lower limb test: $n=12$ strength tests) two level (left and right sides) full factorial (including side $\times$ group interaction) repeated measures multiple analysis of covariance (repeated measures MANCOVA), with age as covariate, were performed. This was performed to evaluate the overall effect of group for strength across the whole kinetic chain for both sides. Effect sizes as eta square $\left(\eta^{2}\right)$, were interpreted according to Cohen [38] as low $<0.04$, medium $\geq 0.04$ to $<0.36$, and large $\geq 0.36$.

Secondly, as a post hoc test for comparing betweengroup differences for each side, an independent measures analysis of covariance [ANCOVA] (most involved vs non-dominant side, least involved vs dominant side) was performed, with age as the covariate. Based on the ageadjusted means, we calculated adjusted standardized 
mean difference (SMD) or effect sizes (ES) by Cohen's $d$ [38] with 95\% CI [39], where $0.2,0.5$ and 0.8 were considered small, moderate and large, respectively. Personal or demographic variables were compared between groups with unadjusted and independent univariate conventional statistics (see Table 1, Results). Alpha was set to 0.05 for all statistical tests (SPSS, v.25, IBM, NY, USA), as no adjustments are needed for multiple comparisons in explorative studies [14-16, 40-42].

\section{Results}

\section{Recruitment result}

Two patients were recruited in physiotherapy clinics, without information on those who declined. At the hospital, we recruited 27 patients out of 36 eligible, where 10 of those invited chose not to participate. The reasons for declining were long traveling distances $(n=3)$, not interested $(n=4)$, afraid of strength testing $(n=2)$, and too time-consuming $(n=1)$. One participant answered the questionnaire but withdrew before the lab-test due to a flare-up at home. This person later withdrew with no stated reason and was excluded from the analysis. Five individuals with KOA were excluded from participation due to old age $(n=3)$, BMI, and an unstable heart. Thus, in total we analyzed/included 28 patients and 31 healthy controls in this study.

We aimed to match the groups on gender and age, however, because of inadequate recruitment in the private physiotherapy setting, we had to change the recruitment into secondary/hospital care with comparably older patients. Then, as the recruitment of healthy subjects was aimed at the working population (due to problems with recruiting unaffiliated healthy early pensioners), we experienced a between-group difference in age that we had to adjust statistically.

\section{Personal and clinical characteristics}

The patients with KOA were on average 6.4 years older (than the controls). There were no other significant differences for personal/demographic factors. On average, the patients had experienced pain for 11 years, and they were diagnosed 10 years ago. Further, they had mostly small to moderate radiographic $\mathrm{KOA}$ and moderate symptoms. Table 1 shows the personal/demographic and non-strength clinical factors.

A significant moderate overall interaction effect of side and group was indicated when all measures for both sides were collapsed into the repeated measures MANCOVA model (Wilks' Lambda $[\mathrm{WL}]=0.825, \mathrm{~F}_{1}$, $\left.{ }_{56}=11.845, \eta^{2}=0.175, P=0.001\right)$. However, then there was no significant main effect for side $\left(\mathrm{WL}=0.997, \mathrm{~F}_{1}\right.$, $\left.{ }_{56}=0.161, \eta^{2}=0.003, P=0.690\right)$ nor an interaction effect between side and age $\left(\mathrm{WL}=0.998, \mathrm{~F}_{1,56}=0.119\right.$, $\eta^{2}=0.002, P=0.731$ ).
A main effect of group showed a near significant difference in total strength for the whole kinetic chain and both sides $\left(\mathrm{F}_{1,56}=3.902, \eta^{2}=0.065, P=0.053\right)$ indicating a strength deficit in the KOA group.

The post hoc test between-group ANCOVA, however, showed significant muscle weakness of moderate magnitude in six joint-strength directions on the (most) involved side in patients with KOA compared to controls. Specifically, the most substantial muscle weaknesses were found in hip internal rotation and ankle eversion, and hip external rotation. Further, still moderate but somewhat smaller weaknesses were found in knee extension and ankle dorsal flexion and inversion on the most involved side. The only significant weakness finding on the (least or) uninvolved leg was for ankle dorsal flexion (Table 2). There were no significant differences between groups for 17 of the 24 strength tests.

Figure 1 indicates the relative difference in muscle weakness across legs and joint-strength directions in the patients with KOA compared to healthy individuals.

\section{Discussion \\ Principle findings}

Overall, across legs and join-strength directions, the most substantial muscle weakness were found in the involved leg for the muscles that evert and invert the ankle (i.e., that effect mainly frontal-plane shank-foot-ground interactions during gait) and for muscles that internally and externally rotate the femur at the hip (i.e., that effect mainly transversal-plane pelvic-femur interactions). Still further on the involved side, we found about the same magnitude of weakness for the muscles that extend the knee and plantar and dorsal flex the ankle (i.e., that effect mainly sagittal-plane femoral-shank-foot-ground interactions). There were no significant differences for the remaining $67 \%$ of the tests. This is the first study that has comprehensively explored muscle strength across the whole kinetic chain of the lower extremities bilaterally in patients with KOA versus healthy controls.

\section{Results discussion}

The current finding of most substantial hip external rotation weakness, is fairly concurrent with three other case-control studies [43-45]. Our ad hoc meta-analysis of these (studies) showed a large between-group difference (30\% in mean; ES 0.9, 95\% CI 0.4 to 1.37 ) based on 163 cases and 97 controls. Further, their gender distributions agreed with that seen in our study. Because there are now three studies with similar point estimated and variable discriminative findings, adding more studies with the same small-to-moderate severely affected KOA population might not change this evidence. As for clinical trial evidence, a recent systematic review [11] of randomized control trials (RCTs) demonstrated large 
Table 2 Strength differences between patients with KOA and control individuals without knee complaints unadjusted and adjusted for age

\begin{tabular}{|c|c|c|c|c|c|c|c|c|c|c|}
\hline \multirow{3}{*}{$R_{n}$} & \multirow{3}{*}{$\begin{array}{l}\text { Strength } \\
\text { Joint Dir }\end{array}$} & \multirow{2}{*}{\multicolumn{2}{|c|}{$\frac{\text { KOA-group }}{\mathrm{M}(\mathrm{SD}) \text { [in Nm/kg unit] }}$}} & \multicolumn{2}{|l|}{ HC-group } & \multirow{2}{*}{\multicolumn{2}{|c|}{ SMD }} & \multicolumn{3}{|c|}{$\mathrm{KOA}-\mathrm{HC}$ group } \\
\hline & & & & & & & & \multirow{2}{*}{$\begin{array}{l}\text { M \% d } \\
- \text { Adj }\end{array}$} & \multicolumn{2}{|l|}{$P$-values } \\
\hline & & Unadj (SD) & Adj M (SD) & Unadj M (SD) & Adj & Unadj & Adj $(95 \% \mathrm{Cl})$ & & Unadj & Adj \\
\hline 1 & Hip IR I & $0.74(0.27)$ & $0.75(0.25)$ & $0.96(0.21)$ & $0.94(0.25)$ & -0.9 & $-0.7(-1.3,-0.2)$ & 22.5 & 0.0009 & 0.0092 \\
\hline 2 & Ankle EV I & $0.19(0.08)$ & $0.20(0.08)$ & $0.27(0.07)$ & $0.26(0.08)$ & -1.0 & $-0.7(-1.3,-0.2)$ & 26.1 & 0.0006 & $0.0096 \dagger$ \\
\hline 3 & Hip ER I & $0.25(0.10)$ & $0.26(0.12)$ & $0.36(0.13)$ & $0.35(0.12)$ & -0.9 & $-0.7(-1.2,-0.2)$ & 29.5 & 0.0008 & $0.013^{*}$ \\
\hline 4 & Knee EXT I & $1.16(0.48)$ & $1.15(0.46)$ & $1.46(0.38)$ & $1.48(0.46)$ & -0.7 & $-0.7(-1.2,-0.2)$ & 25.1 & 0.0103 & $0.012^{*}$ \\
\hline 5 & Ankle PF I & $0.56(0.24)$ & $0.58(0.25)$ & $0.75(0.23)$ & $0.73(0.25)$ & -0.5 & $-0.6(-1.2,-0.1)$ & 22.9 & 0.0029 & 0.073 \\
\hline 6 & Ankle DF I & $0.17(0.07)$ & $0.17(0.08)$ & $0.23(0.09)$ & $0.22(0.08)$ & -0.8 & $-0.6(-1.1,-0.1)$ & 25.6 & 0.0043 & $0.025^{*}$ \\
\hline 7 & Ankle INV I & $0.24(0.10)$ & $0.25(0.10)$ & $0.31(0.08)$ & $0.31(0.10)$ & -0.8 & $-0.6(-1.1,-0.1)$ & 21.4 & 0.0055 & $0.033^{*}$ \\
\hline 8 & Hip AD U & $0.75(0.33)$ & $0.76(0.31)$ & $0.93(0.25)$ & $0.92(0.31)$ & -0.6 & $-0.5(-1.0,0.0)$ & 19.0 & 0.0227 & 0.057 \\
\hline 9 & Ankle DF U & $0.17(0.06)$ & $0.18(0.08)$ & $0.23(0.08)$ & $0.22(0.08)$ & -0.7 & $-0.5(-1.0,0.0)$ & 20.0 & 0.0096 & $0.021^{*}$ \\
\hline 10 & Hip AD I & $0.71(0.27)$ & $0.72(0.27)$ & $0.86(0.24)$ & $0.85(0.27)$ & -0.6 & $-0.5(-1.0,0.0)$ & 16.6 & 0.0272 & 0.057 \\
\hline 11 & Knee FLX I & $0.59(0.35)$ & $0.61(0.33)$ & $0.77(0.28)$ & $0.76(0.0 .33)$ & -0.6 & $-0.5(-1.0,0.1)$ & 21.9 & 0.0325 & 0.114 \\
\hline 12 & Hip FLX I & $1.15(0.29)$ & $1.15(0.32)$ & $1.29(0.31)$ & $1.29(0.32)$ & -0.4 & $-0.4(-1.0,0.1)$ & 11.5 & 0.0749 & 0.256 \\
\hline 13 & Knee EXT U & $1.45(0.36)$ & $1.45(0.44)$ & $1.64(0.46)$ & $1.64(0.44)$ & -0.5 & $-0.4(-0.9,0.1)$ & 11.6 & 0.0877 & 0.120 \\
\hline 14 & Hip EXT I & $1.20(0.52)$ & $1.21(0.52)$ & $1.44(0.45)$ & $1.43(0.51)$ & -0.5 & $-0.4(-0.9,0.1)$ & 16.7 & 0.0701 & 0.135 \\
\hline 15 & Hip AB U & $0.85(0.37)$ & $0.88(0.36)$ & $1.05(0.33)$ & $1.02(0.36)$ & -0.6 & $-0.4(-0.9,-0.1)$ & 14.7 & 0.035 & 0.148 \\
\hline 16 & Ankle PF U & $0.59(0.27)$ & $0.61(0.27)$ & $0.73(0.26)$ & $0.71(0.27)$ & -0.6 & $-0.4(-0.9,0.1)$ & 15.2 & 0.0379 & 0.185 \\
\hline 17 & Hip FLX U & $1.19(0.30)$ & $1.19(0.35)$ & $1.30(1.30)$ & $1.30(0.35)$ & -0.3 & $-0.3(-0.8,0.2)$ & 8.8 & 0.2043 & 0.105 \\
\hline 18 & Hip ER U & $0.29(0.11)$ & $0.30(0.12)$ & $0.34(0.13)$ & $0.33(0.12)$ & -0.5 & $-0.3(-0.8,0.2)$ & 9.5 & 0.0671 & 0.288 \\
\hline 19 & Hip AB I & $0.90(0.35)$ & $0.91(0.34)$ & $1.01(0.29)$ & $1.00(0.34)$ & -0.4 & $-0.3(-0.8,0.2)$ & 9.4 & 0.19092 & 0.148 \\
\hline 20 & Ankle EV U & $0.23(0.08)$ & $0.24(0.09)$ & $0.26(0.08)$ & $0.26(0.08)$ & -0.5 & $-0.2(-0.8,0.3)$ & 8.0 & 0.0911 & 0.375 \\
\hline 21 & Hip EXT U & $1.31(0.51)$ & $1.34(0.54)$ & $1.50(0.51)$ & $1.47(0.54)$ & -0.4 & $-0.2(-0.8,0.3)$ & 9.3 & 0.1499 & 0.146 \\
\hline 22 & Knee FLX U & $0.74(0.38)$ & $0.77(0.39)$ & $0.90(0.37)$ & $0.87(0.39)$ & -0.4 & $-0.2(-0.7,0.3)$ & 12.2 & 0.1042 & 0.390 \\
\hline 23 & Ankle INV U & $0.25(0.09)$ & $0.26(0.08)$ & $0.28(0.07)$ & $0.27(0.08)$ & -0.3 & $-0.2(-0.7,0.3)$ & 3.8 & 0.2252 & 0.534 \\
\hline 24 & Hip IR U & $0.80(0.24)$ & $0.82(0.24)$ & $0.87(0.21)$ & $0.85(0.24)$ & -0.3 & $-0.1(-0.7,0.4)$ & 3.6 & 0.2768 & 0.588 \\
\hline
\end{tabular}

Notes. Statistically significant differences are in bold type. KOA Patients in the knee osteoarthritis group, $H C$ Healthy control group, $R_{n}$ Rank-position for the jointand-torque-direction on muscle weakness (i.e., SMD) in the KOA-group compared to the HC-group, ${ }^{*}=$ significant different, $\dagger=$ highly significant (two-tailed ANCOVA); Unadj Unadjusted, Adj Adjusted for the covariate age, I the (most) involved leg (in KOA-group) or non-dominant leg (in HC-group), $U$ Uninvolved leg or dominant leg (if HC-group), dir. = direction, EV Eversion, ER External rotation, IR Internal rotation, INV Inversion, DF Dorsal flexion, EXT Extension, FLX Flexion, PF Plantar flexion, ER External rotation, $M$ Mean, SD Standard deviation, $C I$ Confidence interval (lower limit, upper limit), SMD Standardized mean difference or Cohen's $\mathrm{d}, p$ P-value. All strength measures are peak strength regardless of range of motion, except for the knee joint (peak strength at $30^{\circ}$ flexion) and hip $A B$ or $\mathrm{AD}$ (peak strength in the anatomical position). Results are normalized for body mass (i.e., $M$ and $S D$ are given as $\mathrm{Nm} / \mathrm{kg}$ )

effects on pain and function of hip strengthening exercises and quadriceps exercises as compared to quadriceps exercises alone. Unfortunately, the methodological quality of these trials was low (i.e., a median PEDro score $<6)$. Interestingly though, none of these trials reported to have strengthened the hip external rotators, only the hip abductors. However, note that the hip abductor exercises in these trials probably indirectly exercised four out of 13 muscles known to externally rotate the hip [46]. Thus, in sum, evidence indicate substantial discriminative value of assessing external rotation strength with a promising but insecure and indirect link to strength exercise therapy improving pain and function for patients with KOA.
Hip internal rotation weakness was reported in two previous case-control studies [43, 44], a between-group difference documented in a meta-analysis [12] to be large (29\% in mean; ES $0.8,95 \%$ CI 0.3 to 1.2 ). That result [12] is in fair agreement with the current study through various differences: The proportion of females [88\% [44], ours 62\%], not reported BMI [43] and higher BMI [44], various positions of measuring, and measurement modes [isometric [43], isokinetic $30^{\circ} / \mathrm{s}$ [44], ours isokinetic $\left.60^{\circ} / \mathrm{s}\right]$. As for clinical effects, however, through two recent systematic reviews $[11,47]$ we found no trials that had specifically targeted the hip internal rotators. However, on pain and function, these reviews showed important indirect effects of exercising the hip internal 


\section{Muscle weakness in patients with KOA}

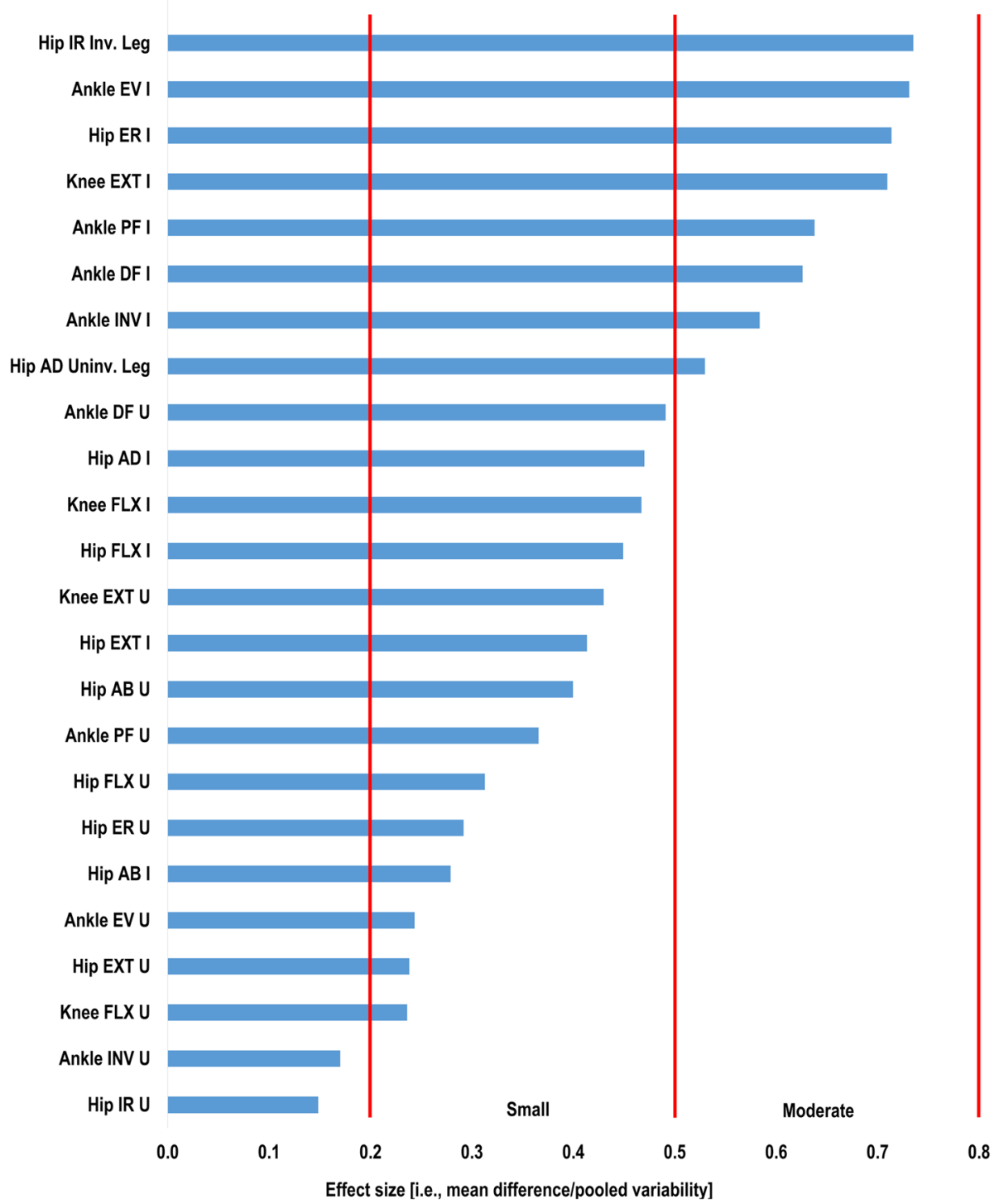

Fig. 1 Muscle weakness as difference between patients with knee osteoarthritis compared to individuals without knee complaints. Strength directions for joints with largest weaknesses on top and the smallest on the bottom. Notes: Effect size = Standardized mean difference or Cohen's $d, \mathrm{KOA}=$ knee osteoarthritis, Inv. $=($ most $)$ involved leg, I $=$ (most) involved leg, EV= eversion, $E R=$ external rotation, FLX = flexion, EXT = extension, $\mathrm{PF}=$ plantar flexion, $\mathrm{DF}=$ dorsal flexion, $\mathrm{INV}=$ inversion, $\mathrm{AD}=$ adduction, Uninv. $=$ (least or) uninvolved leg, $\mathrm{U}=$ (least or) uninvolved leg, $A B=$ abduction

rotators by using programs that applied hip abductor exercises which probably indirectly loaded three out of seven hip internal rotators [46]. Thus, evidence indicate important test discrimination and indirect exercise effect of hip internal rotation strengthening on pain and function in KOA.

Ankle strength is the least examined construct as compared to studies on knee and hip strength. On the one hand, we did not find other case-control data on ankle eversion strength. Such strength is also unreported for healthy individuals according to a recent systematic review [48]. On the other hand, the ankle inversion muscle weakness in the current study is slightly less pronounced than the finding of Park et al. (2016) [45], whom reported a large effect size $(0.84,95 \%$ CI 0.25 to 1.43) of isometric testing presented as $\mathrm{N} / \mathrm{kg}$ (vs ours $\mathrm{Nm} / \mathrm{kg}$ ). Strength, however, is most validly presented as $\mathrm{Nm} / \mathrm{kg}$ [49]. A more important risk of bias in that study [45] appears to be the lack of reporting the exact method of measuring inversion strength. Thus, the above wide confidence interval, low number of studies, and the methodological uncertainty, makes this evidence 
very likely to change with future studies. As for clinical trial effects, we found no prior strength exercise studies having explicitly reported targeting these mainly frontal plane ankle muscles. Thus, in sum, evidence indicates uncertain but substantial discrimination on ankle strength mainly in the frontal plane with an unexplored therapeutic link in KOA.

The knee extension weakness in patients with KOA is large on average. According to a recent meta-analysis of 27 cross-sectional case-control studies [13] whereto we added five more [45, 50-53], the between-group difference amounted $23 \%$ and a large effect size $(0.8,95 \% \mathrm{CI}$ 0.2 to 1.5$)$. The present study's moderate muscle weakness thus falls into the middle to lower range of this confidence interval. Possibly the muscle weakness in the current study could have been more pronounced if our data had been extracted in a more flexed knee position than $30^{\circ}$. Indeed, among the 11 highest ranked studies in our ad hoc meta-analysis, we found large knee extension weakness among all five isokinetic studies [54-58] that recorded peak strength at $54^{\circ}$ of knee flexion on average (our calculation). The large muscle weakness variability in the total meta-analyzed result and the small lower limit of its confidence interval, indicate that the true knee extension weakness does indeed vary largely in this population, a fact that is unlikely to change with future research. On pain and function, the clinical importance of knee extension strength exercises in $\mathrm{KOA}$ is indisputable $[8,59]$.

The current study found moderate weakness in ankle plantar flexion. Previous case-control results were metaanalyzed [45, 50, 52, 60-63] and showed large difference (24\% in mean; ES 0.82, 95\% CI 0.3 to 1.3 ) between 301 cases and 272 controls. Again, this muscle weakness is more substantial than our moderate finding and those studies represent a lower proportion of females (38\% females vs. our 64\%). Further, the mean difference in percentage from the meta-analyzed material ranged from 50 to $1 \%$ (vs ours 19\%) and confidence intervals ranged from small to large. Thus, this evidence is likely to change with future studies, although it might as well indicate a truly large sample variation. Of promising therapeutic importance, the plantar and invertor muscles have indicated a substantial external knee abduction moment via their impact on the ground reaction force during gait [64]. This seems important, due to its possible mitigating effect on a highly prevalent medial radiographic KOA shown to be positively associated with (although unproven to be caused by) an increase in the external knee adduction moment [65]. However, on pain and function, the only evidence of therapeutic effects of ankle plantar flexion exercise appears to be indirect; that is, through trial programs strengthening the kinetic chain through the one-legged press only $[66,67]$. Thus, evidence indicate substantial point discrimination and variability of assessment and indirect exercise effects on pain and function of ankle plantar flexors strength in KOA.

The biomechanical mechanisms of KOA appear to be knee instability and muscle weakness in the frontal [51, 68], transverse [64, 69], and sagittal plane [13]. That is, the mechanisms behind the long-term symptomatic KOA might be selective weakness of the soleus and gastrochnemius [64], the fibularii, the tibialis anterior, the hip internal-external rotators, and the quadriceps muscle [actuating sagittal and frontal plane control [70]]. Here we present recent arguments, starting off in the frontal plane.

A particularly strong cross-sectional case-control study [51] indicate joint instability in the frontal plane and thereto cartilage wear as a plausible injury mechanism. Having applied highly accurate dynamic stereo Xrays and instrumented gait-way analyses in patients with medial KOA, Farrokhi et al. (2016) [51] found significant (i) elevated tibiofemoral contact point excursions and (ii) elevated frontal plane motion, both during the loading response phase of downhill gait. Further, a case-control simulation study based on in vivo biomechanical analysis of horizontal gait in patients with varus misaligned KOA [64], indicated that the soleus and gastrocnemius muscles offered a significant deficit in external knee abduction moment (effected actively via the ground reaction force) in patients with KOA. That is, a deficit capable of explaining the patients' increased external knee adduction moment at its second peak during late stance phase. This second peak was three times higher than that in the control individuals as compared to the first peak (that was mainly caused by gravity). In the same study [64], gluteus medius was the primary contributor to the external knee adduction moment (via the ground reaction force) in both cases and controls (i.e., a normal finding). However, a major limitation of their [64] muscle modelling was not having included the large gluteal muscles as knee-spanning muscles (i.e., the tensor fascia lata, gluteus medius, and gluteus maximus via their common long tendon - the fascia lata/iliotibial band) [71, 72]. The knee-spanning gluteals probably contribute substantially to the internal (i.e., possibly protective) knee abductor moment due to its large cross-sectional area, long tendon, and large moment arm (as compared to that of the quadriceps in the frontal plane [70]). Comparingly, when preparing for the present study, we found no reliable test for knee abduction strength. Further, the tests found reliable for hip abductor strength in patients with KOA didn't apply resistance inferior to the knee joint, and therefore did not include any knee-spanning moment of the gluteal knee abductor muscles. 
Further in the frontal plane, a prospective cohort study [68] biomechanically assessed patients with varus malaligned KOA during gait. Here, Hodges et al. (2016) [68] documented positive correlation between annual loss of medial tibial cartilage volume and (i) greater duration of medial knee muscle (vastus, semimembranosus) coactivation, and (ii) greater duration of medial relative to lateral knee muscle (vastus lateralis, biceps femoris) coactivation. Higher lateral thigh-muscle co-contraction correlated significantly with decreased cartilage loss. A possible explanation for these patients' apparent maladaptive increase in muscular compression across the medial tibiofemoral joint, is that these medial kneespanning muscles are capable of increasing the external knee abduction moment via their (joint-coupled) influence on the ground reaction force [64].

In the transverse plane, in downhill walking - the most problematic activity for patients with KOA [51] most of the deep external rotators of the hip are at short length and thus force-length weakened (due to the slightly flexed-to-extended positions of the hip) [73, 74]. That is, the already weakened external rotators, as tested in lengthened positions in the present study, become even weaker by the downhill-walking hip movement pattern. Further, the external rotators of the hip are documented as the group most vulnerable to muscle weakness during gait [75]. As for the role of muscle weakness of the hip internal rotators, however, we speculate that they have an important cocontracting and hip-stabilizing role in concert with the external rotators, much similarly to that of the hamstring muscles concerting the main knee muscle quadriceps during external knee flexion moment loading in the sagittal plane [70].

Thus, in support of (i) the present study, (ii) strength trial meta-analyses [12, 13], and (iii) in-vivo anchored simulations [64], possible therapeutic solutions might be as follows: To increase the strength of the hip external and internal rotators and knee-spanning hip abductors, the lateral knee extensors and flexors (i.e., the kneespanning knee abductors), and the ankle invertors and plantar flexors (i.e., the non-knee spanning knee abductors). On the core outcomes pain and function, evidence from two systematic reviews of randomized controlled trials [2018] [11, 47] evaluating the effect of hip muscle strength exercises [47], and hip muscle strength exercises in addition to knee extension strength exercises [11], indirectly hints towards such a mechanism in patients with KOA.

\section{Methods discussion}

The current study has its methodological limitations and strengths. On the one hand, we did not manage to level the groups equally on age, and some readers might miss an alpha correction for the multiplicity of testing according to classical statistical texts [76-80]. Further, the results of the peak knee extensor and flexor strength were confined to the $30^{\circ}$ knee position, and the sample size was moderate $[76,77]$. Moreover, there is evidence of relation between reduced strength with increasing radiographic KL-grade of KOA [81] unadjusted for in the present study. Yet further, one may claim that these strength differences are due to malalignment [82]. Finally, one can ask: could not all the current muscle weaknesses be explained by pain [83-86]? On the other hand, this is the only study so far to have comprehensively explored muscle strength in all main joints and directions bilaterally in a single case-control sample for patients with KOA. Further, we statistically adjusted for the difference in age. Supportingly therein, there was no substantive difference in the statistical inferences between the age-adjusted and the unadjusted analysis. The latter fact is understandable, due to the mean in groups being within the same middle-aged maturational category [45-64 years old] (MeSH, PubMed). Thus (therein), the groups were presenting themselves with the similar age-vs-strength decline risk profile. Concordantly, our findings (adjusted or unadjusted) were well aligned with those from appropriately age-matched confirmatory case-control studies. Indeed, in the present study we generally found less pronounced betweengroup differences than what was found in prior studies summarized in meta-analyses thus contradicting an alleged age bias. Further, the explorative nature of this study justifies its main findings by highly significant differences, and corrections for multiple comparisons are judged by reputable statisticians not to be needed in exploratory studies [14-16, 40-42]. Yet further, our peak knee extension strength position of $30^{\circ}$ adds valuable data compared to the average peak strength position of $54^{\circ}$ of prior isokinetic case-control studies [13]. Moreover, there is way more evidence against an association between radiographic grade of KOA and strength [82, $87,88]$ than the indirect association found for it in a single cohort [81]. Yet further, there is systematic review and meta-analysis evidence against the association between $\mathrm{KOA}$ and malalignment [13]. Even further, although several studies show an association between increased pain and decreased strength (chiefly in the knee extensor muscles), there exists opposing evidence [83, 89-91]. More importantly thereto, the current study was not designed to build a strong presumably causative or associative claim as to why these patients were weaker in all these muscle-groups. Thus, we infer adequate internal validity of the current study.

The extensiveness of our testing of muscle groups in the lower limb is limited by excluding the toe flexor muscles [92-94]. Additionally, the external validity of 
the study is limited to patients below 70 years of age and BMI obesity class I (excluding WHO's obesity grade IIIII). Furthermore, because the current sample size was moderate and the study exploratory designed [14, 15], we acknowledge the need for larger exploratory and confirmatory studies to further substantiate the present findings. Still, we infer the current study to be appropriately externally valid.

\section{Potential clinical research implications}

What might be the possible clinical research implications of the evidence analysis above? In order to improve pain and function, clinical researchers may apply ours and others' case-control findings, together with metaanalytic trial evidence $[11,12]$, to incorporate strengthening of weak ankle and hip muscles into the existing so called "hip abductor exercises" $[95,96]$ together with a simple and effective [97] open chain quadriceps program [98]. Then all this can be compared to a control group given the latter active quadriceps exercise program [98] only. The first protocol is hypothesized to account for the possibility that the most important muscles for an apparent knee cartilage protecting internal knee abduction moment $[65,68]$ might be the quadriceps [70] and the knee-spanning gluteal muscles [64, 71, 99]. Interestingly, these latter knee-spanning gluteals, together with the hip external rotators [73] and the ankle evertors, are probably all strengthened in the promising standing hipflexed wall abduction exercise described in Ashok's recent RCT [95]. Interesting indeed, because, according to a systematic review and meta-analysis of RCTs [11], that particular exercise is described in the most effective experimental program on pain and function as compared to an active quadriceps control-exercise group in the Ashok (2012) trial [95].

\section{Conclusions}

Conclusively, this exploratory study indicates that the most substantial muscle weaknesses are in the involved leg's hip and ankle muscles with main actions in the frontal and transverse planes of the kinetic chain of importance for gait. Slightly less substantial, it still indicates important weakness of the knee extensor muscles. That is, in patients aged 45 to 70 years with knee osteoarthritis with light-to-moderate disease severity in a primary/hospital care setting. Future confirmative studies are needed to evaluate the validity and clinical relevance of these findings. Clinical trialists are suggested to build on existing strength programs that already include these ankle and hip muscle-groups in addition to the knee extensor muscles, and that appear highly effective on pain and function according to a metaanalysis of randomized controlled trials.

\section{Abbreviations}

6MWT: Six-minute walk distance test; ACR: American College of

Rheumatology; ANCOVA: Analysis of covariance; BMI: Body mass index; Cl: Confidence interval; ES: Effect size; EULAR: European League Against Rheumatism; FUNKART: the mechanism for function with knee osteoarthritis study; GP: General physician; HHD: Hand held dynamometer; KOA: Knee osteoarthritis; KOOS: Knee Injury and Osteoarthritis Outcome Score; MANCOVA: Multiple analysis of covariance; N: Newton; NIST: National Institute of Standards and Technology; Nm: Newton meter;

OA: Osteoarthritis; PEDro: Physiotherapy Evidence Database; P-P plots: Probability-probability plot; RCT: Randomized controlled trial; REC: Regional Ethics Committee; SMD: Standardized mean difference; T10StUpDwT: Timed 10-Step Up and Down Stair Climb Test

\section{Acknowledgements}

We thank the participants for time and effort, our scientific-assistants, Erik Borg Kolsung and Anja Liljegren, for co-developing and implementing our Biodex protocol and test-assistance, the student-assistant Tina Marlen Bråten Mella for the latter, the training- and testing-expert, Dale Reese, for practical Biodex training, and professor Karin Roeleveld for support with analysis of Biodex data. We also thank the engineers Per Bendik Wik and Xiangchun Tan at NeXt Move Core Facilities, NTNU, for advices regarding strength testing and analysis.

\section{Authors' contributions}

All authors substantially revised the manuscript versions for clinical and scientific content and approved the submitted version in the present form (as well as the versions leading up to it). Further, all authors have agreed to be personally accountable for the author's own contributions and to ensure that questions related to the accuracy or integrity of any part of the work, even ones in which the author was not personally involved, are appropriately investigated, resolved, and the resolution publicly documented. $\mathrm{KV}$, AKS, and KGN have substantially contributed to the conception and design of the work. I.e., AKS secured funding for the project, KV wrote the research proposal update, ethical application, and protocol, and KGN substantially revised these documents together with AKS. KV and ME extensively recruited participants and collected data. KV and HL substantially analyzed and interpreted data. KV drafted and redrafted all manuscript versions before and between rounds of revisions. $\mathrm{KV}$ is the guarantor of the manuscript's authenticity.

\section{Funding}

This project received internal funding from the former Sor-Trondelag University College for a PhD-student position. The funding was granted to AKS, the head of the study titled "Mechanisms for improved physical function with knee osteoarthritis". There exists no grant number. The funders had no role in study design, data collection and analysis, decision to publish, nor in writing the manuscript.

\section{Availability of data and materials}

The dataset generated and analyzed during the current study are available on reasonable request from the head of the project AKS or from the Department of Neuroscience and Movement Science, Faculty of Medicine and Health Science, Norwegian University of Science and Technology.

Ethics approval and consent to participate

The FUNKART study was approved by a Regional Ethics Committee for Medical and Health Research (the Regional Ethics Committee North, RECnorth 2016/984 [In Norwegian REK-nord]) and conducted according to the Helsinki declaration and Norwegian laws. The participants signed the approved written consent before participation.

\section{Consent for publication}

Not applicable.

\section{Competing interests}

The authors declare that they have no competing interests regarding the present study. 


\section{Author details}

${ }^{1}$ Norwegian University of Science and Technology, Trondheim, Norway. ${ }^{2}$ Department of Physical Education and Sport Science, Nord University, Levanger, Norway. ${ }^{3}$ Umea University, Surgical and Perioperative Sciences, Umea, Sweden. ${ }^{4}$ Department of Physiotherapy, Clinic of Clinical Services, Trondheim University Hospital, Trondheim, Norway.

Received: 26 July 2019 Accepted: 19 November 2019

Published online: 09 December 2019

\section{References}

1. Vos T, Abajobir AA, Abate KH, Abbafati C, Abbas KM, Abd-Allah F, Abdulkader RS, Abdulle AM, Abebo TA, Abera SF, Aboyans V. Global, regional, and national incidence, prevalence, and years lived with disability for 328 diseases and injuries for 195 countries, 1990-2016: a systematic analysis for the Global Burden of Disease Study 2016. Lancet. 2017; 390(10100):1211-59.

2. Oliveria SA, Felson DT, Reed Jl, Cirillo PA, Walker AM. Incidence of symptomatic hand, hip, and knee osteoarthritis among patients in a health maintenance organization. Arthritis Rheum. 1995;38(8):1134-41.

3. Sundhedsstyrelsen. Knæartrose - nationale kliniske retningslinjer og faglige visitationsretningslinjer. 1.0 ed. Online: Sundhedsstyrelsen; 2012. p. 88.

4. Zhang W, Doherty M, Peat G, Bierma-Zeinstra MA, Arden NK, Bresnihan B, et al. EULAR evidence-based recommendations for the diagnosis of knee osteoarthritis. Ann Rheum Dis. 2010;69(3):483-9.

5. Altman RD. Criteria for the classification of osteoarthritis of the knee and hip. Scand J Rheumatol Suppl. 1987;65:31-9.

6. Roos EM, Arden NK. Strategies for the prevention of knee osteoarthritis. Nat Rev Rheumatol. 2016;12(2):92-101.

7. Thorstensson CA, Andersson ML, Jonsson H, Saxne T, Petersson IF. Natural course of knee osteoarthritis in middle-aged subjects with knee pain: 12year follow-up using clinical and radiographic criteria. Ann Rheum Dis. 2009; 68(12):1890-3

8. Brosseau L, Taki J, Desjardins B, Thevenot O, Fransen M, Wells GA, et al. The Ottawa panel clinical practice guidelines for the management of knee osteoarthritis. Part two: strengthening exercise programs. Clin Rehabil. 2017; 31(5):596-611.

9. Brosseau L, Taki J, Desjardins B, Thevenot O, Fransen M, Wells GA, et al. The Ottawa panel clinical practice guidelines for the management of knee osteoarthritis. Part one: introduction, and mind-body exercise programs. Clin Rehabil. 2017;31(5):582-95.

10. Brosseau L, Taki J, Desjardins B, Thevenot O, Fransen M, Wells GA, et al. The Ottawa panel clinical practice guidelines for the management of knee osteoarthritis. Part three: aerobic exercise programs. Clin Rehabil. 2017;31(5): 612-24.

11. Hislop AC, Collins NJ, Tucker K, et al. Does adding hip exercises to quadriceps exercises result in superior outcomes in pain, function and quality of life for people with knee osteoarthritis? A systematic review and meta-analysis British Journal of Sports Medicine Published Online First: 06 February 2019. https://doi.org/10.1136/bjsports-2018-099683.

12. Deasy M, Leahy E, Semciw Al. Hip Strength Deficits in People With Symptomatic Knee Osteoarthritis: A Systematic Review With Meta-analysis. J Orthop Sports Phys Ther. 2016;46(8):629-39.

13. van Tunen JAC, Dell'Isola A, Juhl C, Dekker J, Steultjens M, Thorlund JB, et al. Association of malalignment, muscular dysfunction, proprioception, laxity and abnormal joint loading with tibiofemoral knee osteoarthritis - a systematic review and meta-analysis. BMC Musculoskelet Disord. 2018;19(1):273.

14. Althouse AD. Adjust for Multiple Comparisons? It's Not That Simple. Ann Thorac Surg. 2016;101(5):1644-5.

15. Bender R, Lange S. Adjusting for multiple testing--when and how? J Clin Epidemiol. 2001;54(4):343-9.

16. Rothman KJ, Greenland S, Lash TL, Buehler JW, Cahill J, Glymour MM, et al. Modern epidemiology. 3rd ed. Philadelphia: Wolters Kluwer/Lippincott Williams \& Wilkins; 2008.

17. Kellgren JH, Lawrence JS. Radiological assessment of osteo-arthrosis. Ann Rheum Dis. 1957;16(4):494-502.

18. de Araujo Ribeiro Alvares JB, Rodrigues R, de Azevedo FR, da Silva BG, Pinto RS, Vaz MA, et al. Inter-machine reliability of the Biodex and Cybex isokinetic dynamometers for knee flexor/extensor isometric, concentric and eccentric tests. Phys Ther Sport. 2015;16(1):59-65.
19. Hartmann A, Knols R, Murer K, de Bruin ED. Reproducibility of an isokinetic strength-testing protocol of the knee and ankle in older adults. Gerontology. 2009;55(3):259-68.

20. Biodex. Biodex Multi-Joint System - Pro Setup/Operation Manual Biodex Medical Systems Inc.; 2014 [Available from: http://www.biodex.com/sites/ default/files/850000man_08262revb.pdf.

21. Baldon RM, Nakagawa TH, Muniz TB, Amorim CF, Maciel CD, Serrao FV. Eccentric hip muscle function in females with and without patellofemoral pain syndrome. J AthlTrain. 2009;44(5):490-6.

22. Thorborg K, Bandholm T, Holmich P. Hip- and knee-strength assessments using a hand-held dynamometer with external belt-fixation are inter-tester reliable. Knee Surg Sports Traumatol Arthrosc. 2013;21(3):550-5.

23. Vaarbakken K, Ljunggren AE. Superior effect of forceful compared to standard traction mobilization in hip disability? Adv Physiother. 2007;9(3): $117-28$.

24. Federation IW. IWF Guidelines Sports Equipment Licensing: International Weightlifting Federation; 2019. p. 19. https://www.iwf.net/wp-content/ uploads/downloads/2015/11/IWF-Guidelines_Sport-Equipment-Licensing.pdf

25. Vaarbakken K, Loras H, Nilsson KG, Engdal M, Stensdotter AK. Relative difference among 27 functional measures in patients with knee osteoarthritis: An exploratory cross-sectional case-control study. BMC Musculoskelet Disord. 2019;20(1):462 In press.

26. Syltebo R, Odegaard TT. InfoPad AS 2012 [Available from: https:/infopad.no/

27. Goldsmith ES, Taylor BC, Greer N, Murdoch M, MacDonald R, McKenzie L, et al. Focused Evidence Review: Psychometric Properties of PatientReported Outcome Measures for Chronic Musculoskeletal Pain. J Gen Intern Med. 2018;33(Suppl 1):61-70.

28. Farrar JT, Young JP Jr, LaMoreaux L, Werth JL, Poole RM. Clinical importance of changes in chronic pain intensity measured on an 11-point numerical pain rating scale. Pain. 2001;94(2):149-58.

29. Dobson F, Hinman RS, Hall M, Marshall CJ, Sayer T, Anderson C, et al. Reliability and measurement error of the Osteoarthritis Research Society International (OARSI) recommended performance-based tests of physical function in people with hip and knee osteoarthritis. Osteoarthr Cartil. 2017 ; 25(11):1792-6.

30. Roos EM, Roos HP, Lohmander LS, Ekdahl C, Beynnon BD. Knee Injury and Osteoarthritis Outcome Score (KOOS)--development of a self-administered outcome measure. J Orthop Sports Phys Ther. 1998;28(2):88-96.

31. Roos EM, Lohmander LS. The Knee injury and Osteoarthritis Outcome Score (KOOS): from joint injury to osteoarthritis. Health Qual Life Outcomes. 2003;1:64

32. McAlindon TE, Bannuru RR, Sullivan MC, Arden NK, Berenbaum F, BiermaZeinstra SM, et al. OARSI guidelines for the non-surgical management of knee osteoarthritis. Osteoarthr Cartil. 2014;22(3):363-88.

33. AbilityLab SR. Rehabilitation Measures Database 2018 [Available from: https:/www.sralab.org/rehabilitation-measures

34. Tveter AT, Dagfinrud H, Moseng T, Holm I. Measuring health-related physical fitness in physiotherapy practice: reliability, validity, and feasibility of clinical field tests and a patient-reported measure. J Orthop Sports Phys Ther. 2014; 44(3):206-16.

35. Dobson F, Hinman RS, Hall M, Terwee CB, Roos EM, Bennell KL. Measurement properties of performance-based measures to assess physical function in hip and knee osteoarthritis: a systematic review. Osteoarthr Cartil. 2012;20(12):1548-62.

36. Trialists'Collaboration SU. Organised inpatient (stroke unit) care for stroke. Cochrane Database Syst Rev. 2013;9(9):Cd000197.

37. Gronhaug G, Osteras N, Hagen KB. Quality of hip and knee osteoarthritis management in primary health care in a Norwegian county: a crosssectional survey. BMC Health Serv Res. 2014;14:598.

38. Cohen J. Statistical power analysis for the behavioral sciences. Hillsdale: Lawrence Erlbaum Associates; 1988.

39. Wilson DB. Practical Meta-Analysis Effect Size Calculator [Online calculator]: Campbell Collaboration; Ph.D. (n.d.) [Available from: https:/www. campbellcollaboration.org/research-resources/research-for-resources/effectsize-calculator.html

40. Rothman KJ. No adjustments are needed for multiple comparisons. Epidemiol (Cambridge, Mass). 1990;1(1):43-6.

41. Perneger TV. What's wrong with Bonferroni adjustments. Bmj. 1998; 316(7139):1236-8,

42. Nelder J. From statistics to statistical science - Reply. J R Stat Soc Ser D-Stat. 1999;48:269. 
43. Hinman RS, Hunt MA, Creaby MW, Wrigley TV, McManus FJ, Bennell KL. Hip muscle weakness in individuals with medial knee osteoarthritis. Arthritis Care Res. 2010;62(8):1190-3.

44. Costa RA, Oliveira LM, Watanabe SH, Jones A, Natour J. Isokinetic assessment of the hip muscles in patients with osteoarthritis of the knee. Clin (Sao Paulo, Brazil). 2010;65(12):1253-9.

45. Park SK, Kobsar D, Ferber R. Relationship between lower limb muscle strength, self-reported pain and function, and frontal plane gait kinematics in knee osteoarthritis. Clin Biomech (Bristol, Avon). 2016;38:68-74.

46. Neumann DA. Kinesiology of the hip: a focus on muscular actions. J Orthop Sports Phys Ther. 2010;40(2):82-94.

47. Neelapala YR, Bhagat M, Shah P. Hip Muscle Strengthening for Knee Osteoarthritis: A Systematic Review of Literature. J Geriatr Phys Ther. 2001; 2018:1.

48. Benfica PDA, Aguiar LT, Brito SAF, Bernardino LHN, Teixeira-Salmela LF, Faria C. Reference values for muscle strength: a systematic review with a descriptive meta-analysis. Br J Phys Ther. 2018;22(5):355

49. American College of Sports Medicine position stand. Progression models in resistance training for healthy adults. Med Sci Sports Exerc. 2009;41(3):687-708.

50. Rutherford DJ, Hubley-Kozey CL, Stanish WD. Changes in knee joint muscle activation patterns during walking associated with increased structural severity in knee osteoarthritis. J Electromyogr Kinesiol. 2013;23(3):704-11.

51. Farrokhi S, Voycheck CA, Gustafson JA, Fitzgerald GK, Tashman S. Knee joint contact mechanics during downhill gait and its relationship with varus/ valgus motion and muscle strength in patients with knee osteoarthritis. Knee. 2016;23(1):49-56.

52. Hubley-Kozey CL, Deluzio KJ, Landry SC, McNutt JS, Stanish WD. Neuromuscular alterations during walking in persons with moderate knee osteoarthritis. J Electromyogr Kinesiol. 2006;16(4):365-78.

53. Yamada H, Koshino T, Sakai N, Saito T. Hip adductor muscle strength in patients with varus deformed knee. Clin Orthop Relat Res. 2001;386:179-85.

54. Wu SH, Chu NK, Liu YC, Chen CK, Tang SF, Cheng CK. Relationship between the EMG ratio of muscle activation and bony structure in osteoarthritic knee patients with and without patellar malalignment. J Rehabil Med. 2008;40(5): 381-6.

55. Baert IA, Jonkers I, Staes F, Luyten FP, Truijen S, Verschueren SM. Gait characteristics and lower limb muscle strength in women with early and established knee osteoarthritis. Clin Biomech (Bristol, Avon). 2013;28(1):40-7.

56. Baert IA, Mahmoudian A, Nieuwenhuys A, Jonkers I, Staes F, Luyten FP, et al. Proprioceptive accuracy in women with early and established knee osteoarthritis and its relation to functional ability, postural control, and muscle strength. Clin Rheumatol. 2013;32(9):1365-74.

57. Cheing GL, Hui-Chan CW. The motor dysfunction of patients with knee osteoarthritis in a Chinese population. Arthritis Rheum. 2001;45(1):62-8.

58. Kumar D, Karampinos DC, MacLeod TD, Lin W, Nardo L, Li X, et al. Quadriceps intramuscular fat fraction rather than muscle size is associated with knee osteoarthritis. Osteoarthr Cartil. 2014;22(2):226-34.

59. Fransen M, McConnell S, Harmer AR, Van der Esch M, Simic M, Bennell KL. Exercise for osteoarthritis of the knee: a Cochrane systematic review. $\mathrm{Br}$ J Sports Med. 2015;49(24):1554-7.

60. Elbaz A, Magram-Flohr I, Segal G, Mor A, Debi R, Kalichman L. Association Between Knee Osteoarthritis and Functional Changes in Ankle Joint and Achilles Tendon. J Foot Ankle Surg. 2017;56(2):238-41.

61. Bremander $A B$, Dahl LL, Roos EM. Validity and reliability of functional performance tests in meniscectomized patients with or without knee osteoarthritis. Scand J Med Sci Sports. 2007;17(2):120-7.

62. Goncalves GH, Sendin FA, da Silva Serrao PRM, Selistre LFA, Petrella M

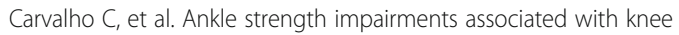
osteoarthritis. Clin Biomech (Bristol, Avon). 2017:46:33-9.

63. Draz AH, Abdel-Aziem AA. Isokinetic assessment of ankle dorsiflexors and plantarflexors strength in patients with knee osteoarthritis. Int Musculoskelet Med. 2015;37(4):164-9.

64. Sritharan P, Lin YC, Richardson SE, Crossley KM, Birmingham TB, Pandy MG Lower-limb muscle function during gait in varus mal-aligned osteoarthritis patients. J Orthop Res. 2018;36(8):2157.

65. Henriksen M, Creaby MW, Lund H, Juhl C, Christensen R. Is there a causal link between knee loading and knee osteoarthritis progression? A systematic review and meta-analysis of cohort studies and randomised trials. BMJ Open. 2014;4(7):e005368.

66. Jan MH, Lin CH, Lin YF, Lin JJ, Lin DH. Effects of weight-bearing versus nonweight-bearing exercise on function, walking speed, and position sense in participants with knee osteoarthritis: a randomized controlled trial. Arch Phys Med Rehabil. 2009;90(6):897-904.

67. Jan MH, Lin JJ, Liau JJ, Lin YF, Lin DH. Investigation of clinical effects of high- and low-resistance training for patients with knee osteoarthritis: a randomized controlled trial. Phys Ther. 2008;88(4):427-36.

68. Hodges PW, van den Hoorn W, Wrigley TV, Hinman RS, Bowles KA, Cicuttini $F$, et al. Increased duration of co-contraction of medial knee muscles is associated with greater progression of knee osteoarthritis. Man Ther. 2016; 21:151-8.

69. Sritharan P, Lin YC, Pandy MG. Muscles that do not cross the knee contribute to the knee adduction moment and tibiofemoral compartment loading during gait. J Orthop Res. 2012;30(10):1586-95.

70. Lloyd DG, Buchanan TS, Besier TF. Neuromuscular biomechanical modeling to understand knee ligament loading. Med Sci Sports Exerc. 2005;37(11): 1939-47.

71. Flack NA, Nicholson HD, Woodley SJ. The anatomy of the hip abductor muscles. Clin Anat (New York, NY). 2014;27(2):241-53.

72. Blemker SS, Delp SL. Three-dimensional representation of complex muscle architectures and geometries. Ann Biomed Eng. 2005;33(5):661-73.

73. Vaarbakken K, Steen H, Samuelsen G, Dahl HA, Leergaard TB, Nordsletten L, et al. Lengths of the external hip rotators in mobilized cadavers indicate the quadriceps coxa as a primary abductor and extensor of the flexed hip. Clin Biomech. 2014;29(7):794-802.

74. Vaarbakken K, Steen H, Samuelsen G, Dahl HA, Leergaard TB, Stuge B. Primary functions of the quadratus femoris and obturator externus muscles indicated from lengths and moment arms measured in mobilized cadavers. Clin Biomech. 2015;30(3):231-7.

75. van der Krogt MM, Delp SL, Schwartz MH. How robust is human gait to muscle weakness? Gait Posture. 2012;36(1):113-9.

76. Field A. Discovering statistics using IBM SPSS statistics : and sex and drugs and rock ' $n$ ' roll. 4th ed. Los Angeles: SAGE; 2013.

77. Altman DG. Practical statistics for medical research, vol. 1991. London: Chapman and Hall; 1991

78. Tukey JW. Some thoughts on clinical trials, especially problems of multiplicity. Science (New York, NY). 1977;198(4318):679-84.

79. Bland JM, Altman DG. Multiple significance tests: the Bonferroni method. Bmj. 1995;310(6973):170.

80. Ludbrook J. Multiple comparison procedures updated. Clin Exp Pharmacol Physiol. 1998;25(12):1032-7.

81. Wada O, Kurita N, Kamitani T, Nakano N, Mizuno K. Influence of the severity of knee osteoarthritis on the association between leg muscle mass and quadriceps strength: the SPSS-OK study. Clin Rheumatol. 2019;38(3):719-25.

82. Lim BW, Hinman RS, Wrigley TV, Bennell KL. Varus malalignment and its association with impairments and functional limitations in medial knee osteoarthritis. Arthritis Rheum. 2008;59(7):935-42.

83. Glass NA, Torner JC, Frey Law LA, Wang K, Yang T, Nevitt MC, et al. The relationship between quadriceps muscle weakness and worsening of knee pain in the MOST cohort: a 5-year longitudinal study. Osteoarthr Cartil. 2013; 21(9):1154-9.

84. Henriksen M, Rosager S, Aaboe J, Graven-Nielsen T, Bliddal H. Experimental knee pain reduces muscle strength. J Pain. 2011;12(4):460-7.

85. Kim D, Park G, Kuo LT, Park W. The effects of pain on quadriceps strength, joint proprioception and dynamic balance among women aged 65 to 75 years with knee osteoarthritis. BMC Geriatr. 2018;18(1):245.

86. Ruhdorfer A, Wirth W, Eckstein F. Association of knee pain with a reduction in thigh muscle strength - a cross-sectional analysis including 4553 osteoarthritis initiative participants. Osteoarthr Cartil. 2017;25(5):658-66.

87. Dell'isola A, Wirth W, Steultjens M, Eckstein F, Culvenor AG. Knee extensor muscle weakness and radiographic knee osteoarthritis progression. Acta Orthop. 2018;89(4):406-11.

88. Whittaker JL, Toomey CM, Woodhouse LJ, Jaremko JL, Nettel-Aguirre A, Emery CA. Association between MRI-defined osteoarthritis, pain, function and strength 3-10 years following knee joint injury in youth sport. Br J Sports Med. 2018;52(14):934-9.

89. Baert IA, Staes F, Truijen S, Mahmoudian A, Noppe N, Vanderschueren G, et al. Weak associations between structural changes on MRI and symptoms, function and muscle strength in relation to knee osteoarthritis. Knee Surg Sports Traumatol Arthrosc. 2014;22(9):2013-25.

90. Riddle DL, Stratford PW. Impact of pain reported during isometric quadriceps muscle strength testing in people with knee pain: data from the osteoarthritis initiative. Phys Ther. 2011;91(10):1478-89. 
91. Sattler M, Dannhauer T, Hudelmaier M, Wirth W, Sanger AM, Kwoh CK, et al. Side differences of thigh muscle cross-sectional areas and maximal isometric muscle force in bilateral knees with the same radiographic disease stage, but unilateral frequent pain - data from the osteoarthritis initiative. Osteoarthr Cartil. 2012;20(6):532-40.

92. Uritani D, Fukumoto T, Myodo T, Fujikawa K, Usui M, Tatara D. The association between toe grip strength and osteoarthritis of the knee in Japanese women: A multicenter cross-sectional study. PLoS One. 2017; 12(10):e0186454

93. Uritani D, Fukumoto T, Matsumoto D, Shima M. The Relationship Between Toe Grip Strength and Dynamic Balance or Functional Mobility Among Community-Dwelling Japanese Older Adults: A Cross-Sectional Study. J Aging Phys Act. 2016;24(3):459-64.

94. Tsuyuguchi R, Kurose S, Seto T, Takao N, Tagashira S, Tsutsumi H, et al. Toe grip strength in middle-aged individuals as a risk factor for falls. J Sports Med Phys Fitness. 2018;58(9):1325-30.

95. Ashok C. Effects of hip abductor muscle strengthening exercises in patients with osteoarthritic knee joints. Indian J Physiother Occup Ther. 2012;6:5.

96. Singh S, Pattnaik M, Mohanty P, Ganesh GS. Effectiveness of hip abductor strengthening on health status, strength, endurance and six minute walk test in participants with medial compartment symptomatic knee osteoarthritis. J Back Musculoskelet Rehab. 2016;29(1):65-75.

97. Young JL, Rhon DI, Cleland JA, Snodgrass SJ. The Influence of Exercise Dosing on Outcomes in Patients With Knee Disorders: A Systematic Review. J Orthop Sports Phys Ther. 2018;48(3):146-61.

98. Huang MH, Lin YS, Yang RC, Lee CL. A comparison of various therapeutic exercises on the functional status of patients with knee osteoarthritis. Semin Arthritis Rheum. 2003;32(6):398-406.

99. Saxby DJ, Modenese L, Bryant AL, Gerus P, Killen B, Fortin K, et al. Tibiofemoral contact forces during walking, running and sidestepping. Gait Posture. 2016;49:78-85.

\section{Publisher's Note}

Springer Nature remains neutral with regard to jurisdictional claims in published maps and institutional affiliations.

Ready to submit your research? Choose BMC and benefit from:

- fast, convenient online submission

- thorough peer review by experienced researchers in your field

- rapid publication on acceptance

- support for research data, including large and complex data types

- gold Open Access which fosters wider collaboration and increased citations

- maximum visibility for your research: over $100 \mathrm{M}$ website views per year

At $\mathrm{BMC}$, research is always in progress.

Learn more biomedcentral.com/submissions 